\title{
Interaction entre des anneaux cohérents de vorticité et la turbulence
}

\author{
Jean-François Krawczynski ${ }^{1}$, Luminata Danaila ${ }^{1, a}$, Bruno Renou ${ }^{1}$ et Paul Dimotakis ${ }^{2}$ \\ ${ }^{1}$ CORIA, UMR 6614, avenue de l'Université, BP 12, 76801 Saint Étienne du Rouvray, France \\ 2 Caltech, Pasadena, USA
}

Reçu le $1^{\text {er }}$ septembre 2006, accepté le 23 janvier 2007

\begin{abstract}
Résumé - Dans un réacteur partiellement mélangé (PaSR, i.e. Partially Stirred Reactor), le fluide est injecté par 16 paires de jets opposés et évacué par des parois poreuses. Ce forçage particulier conduit à la création de paires d'anneaux cohérents de vorticité, tournant dans des sens alternativement opposés. Les propriétés du champ moyen de vorticité et étirement, conduisent à une turbulence en rotation locale, quasi-2D aux grandes échelles. Dans ces anneaux, le champ turbulent est localement homogène et le champ de vorticité reste spatialement corrélé sur des échelles de la taille des anneaux. Les fonctions de structure de vorticité ne présentent pas de lois d'échelles dans la zone inertielle, mais plutôt un comportement en $\log (r)$ ( $r$ est la séparation entre deux points de l'espace), analogue à la turbulence 2D [1]. Afin de décrire le comportement de l'enstrophie sur une large gamme d'échelles, une approche analytique similaire à celle développée par [1] est abordée dans notre cas.
\end{abstract}

Mots clés : Turbulence / jets / rotation

\begin{abstract}
Effect of vorticity coherent rings on turbulence. In a partially stirred reactor (PaSR), fluid is injected through a set of 16 pairs of opposed jets and ejected via porous top and bottom walls. This particular manner of fluid circulation allows for creating pairs of alternating-rotating large-scale coherent rings. The large-scale properties of the mean flow, i.e. large-scale vorticity and strain, lead to a turbulence in local rotation (quasi-2D) at large scales. In these giant structures, turbulent field is locally homogeneous and vorticity field is spatially correlated over a range of scales as large as the rings size. Inertial range of enstrophy second-order structure functions behaves as $\log (r)$ ( $r$ is the separation between two space points), as it is the case for 2D turbulence [1]. In order to describe the enstrophy over the whole range of scales $r$, an analytical approach similar to that adopted in [1] is developed in our case.
\end{abstract}

Key words: Turbulence / jets / rotation

\section{Introduction}

Ce travail est intégré dans un effort de longue durée, qui concerne l'amélioration de nos connaissances sur le mélange turbulent, pour un grand spectre d'applications, incluant la combustion, la propulsion, l'industrie chimique. Des méthodes traditionnelles destinées à obtenir un «bon mélange » sont souvent reliées aux réacteurs $([2-4])$. Des hélices ou des « HEV $»$ (high efficiency vortex) sont également utilisés afin de mieux mélanger. Pourtant, cet aspect est souvent abordé dans des revues de chimie, éludant le point de vue sur la physique du micro-mélange. Le but de cette contribution est d'étudier les propriétés de la turbulence, lorsque la

\footnotetext{
a Auteur correspondant : danaila@coria.fr
}

vorticité est injectée sur une large gamme d'échelles, c'està-dire par paires d'anneaux cohérents, tournant dans des directions alternativement opposées. Nous montrons que cette manière originale d'injecter l'énergie et l'enstrophie conduit à des propriétés particulières de l'écoulement.

Dans cet article, nous montrons que les interactions non-locales dans la cascade d'enstrophie jouent un rôle primordial. Plus précisément, nous suggérons que les statistiques de la vorticité peuvent être expliquées en prenant en compte l'effet de la vorticité du champ moyen, sur les échelles plus petites. Les corrélations en deux points de la vorticité sont principalement influencées par les échelles les plus grandes, qui peuvent être décrites en utilisant le tenseur des dérivées de la vitesse moyenne, avec une partie symétrique (étirement) et une partie anti-symétrique (vorticité). Ceci est en accord avec l'image classique de 

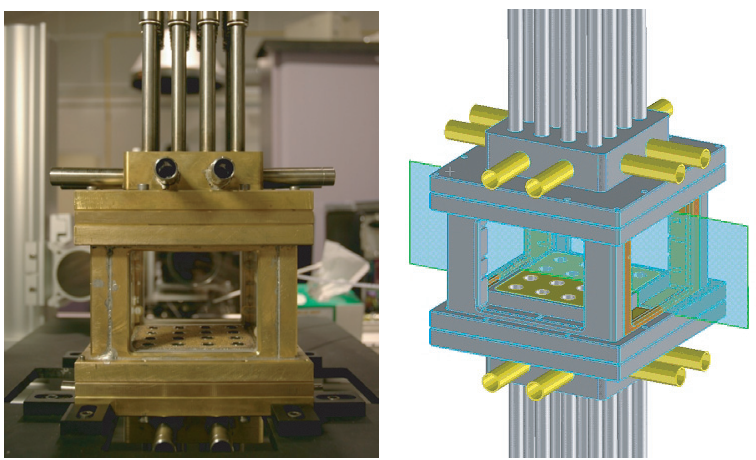

Fig. 1. Installation expérimentale (gauche) et exemple de plan de mesure (droite).

cascade d'enstrophie : un blob de fluide entraîné dans un champ de vorticité grandes-échelles est allongé selon la/les direction(s) d'étirement et comprimé selon la/les direction(s) de compression. Un tel étirement conduit à un flux d'enstrophie vers les plus petites échelles. La vorticité fait tourner le blob de fluide, en diminuant l'étirement, à cause du changement des axes d'étirement positif et négatif.

La section 2 est dédiée à la description de l'installation expérimentale et de l'écoulement. L'analyse spectrale (Sect. 3.1) sera suivie par un diagnostic des flux d'énergie et d'enstrophie à travers les échelles (Sect. 3.2). Notre question suivante concerne le comportement qualitatif en fonction de l'échelle, de l'enstrophie, et est adressée dans la section 3.3. Les conclusions sont tirées dans la section 4.

\section{Installation expérimentale et description de l'écoulement}

L'installation expérimentale (Fig. 1) est un réacteur partiellement mélangé avec des conditions de fonctionnement variables. Le fluide est injecté par 16 paires de jets opposés qui sortent de la partie haute et basse du réacteur et produisent un plan de stagnation (en moyenne) dans le centre du PaSR, où la vitesse moyenne est presque zéro en chaque point. Le diamètre des jets d'injection est $d=1 \mathrm{~cm}$. Le volume du réacteur est de $V=11 \times 11 \times 6 \mathrm{~cm}^{3}$. Sa hauteur peut être modifiée en utilisant des espaceurs. Le fluide sort à travers des plaques poreuses situées sur les plans d'injection. Afin de faire varier le nombre de Reynolds sur une large gamme, la pression du fluide dans le réacteur est modifiable entre [1-3] atm. Les parois latérales sont transparentes, permettant un accès optique pour les mesures de vitesse $2 \mathrm{D}$.

Dans la région des jets, l'axisymétrie locale est bien respectée. Les caractéristiques de l'écoulement sont : - débit de l'écoulement : $Q_{v}=[60-150] \mathrm{m}^{3} \cdot \mathrm{h}^{-1}$; - vitesse d'injection pour chaque jet $\in[6,6-17] \mathrm{m} . \mathrm{s}^{-1}$; - nombre de Reynolds basé sur la micro-échelle de Taylor $R_{\lambda} \in[60-1200]$ (le maximum étant atteint dans la région centrale du réacteur).

Les mesures sont faites en utilisant un algorithme PIV multi-pass adaptatif (le package standard commercial
Davis 6.2, LaVision). La taille finale de la fenêtre d'interrogation est de 16 pixels $^{2}$, avec un recouvrement de $50 \%$. Le grandissement est de 0,038 mm.pixels ${ }^{-1}$. Ainsi, la plus petite échelle résolue est d'approximativement $8 \eta$. Des moyennes temporelles sont effectuées en chaque point de l'image, sur 1500 images.

La figure 2 illustre les composantes de la vitesse moyenne dans le plan des jets : selon la direction horizontale $X$ et selon la direction de l'injection (verticale) $Z$, $V_{Z}$. Ici, $X, Y$ et $Z$ représentent le repère fixe (du laboratoire). L'axisymétrie de l'écoulement autour de l'axe des jets opposés nous permet d'utiliser les coordonnées cylindriques $(R, \theta, z)$ (indiquées sur les figures pour chaque paire de jets). Le plan central de stagnation est à noter. Dans ce plan, toutefois, le fluide a une vitesse moyenne non-nulle selon les directions normales $(R$ et $\theta)$. Dans la zone située entre l'injection par des jets et le retour par des plaques poreuses, il est à noter la présence de couches de mélange à contre-courant axisymmétriques. Cette remarque est particulièrement importante pour la suite du travail. Dans ces régions, la turbulence est en cisaillement pur, avec le cisaillement variable d'un point à l'autre de l'écoulement. Nous parlerons d'une injection d'énergie et d'enstrophie multi-échelles.

La figure 3 représente les distributions des valeurs $R M S$ des vitesses fluctuantes $u_{R}$ et $u_{Z}$. Une bonne isotropie globale de la zone étudiée est à observer, c'est-à-dire que les moyennes spatiales de ces valeurs $R M S$ restent comparables, à $25 \%$ près. Toutefois, les valeurs locales sont différentes et elles délivrent une information sur la structure locale de l'écoulement. Ainsi, de fortes fluctuations de $u_{Z}$ apparaissent devant les jets opposés, alors que des valeurs importantes des fluctuations de $u_{R}$ sont présentes dans le point central, entre les paires de jets. Nous devons clairement souligner ici que notre écoulement est $3 \mathrm{D}$, car la vitesse fluctue selon les trois directions de l'espace.

La figure 4 illustre la présence d'une forte énergie cinétique dans la zone d'impact entre les jets opposés, alors qu'elle devient nettement plus faible dans les zones d'injection.

La vorticité de l'écoulement moyen, $\vec{\Omega} \equiv$ $\left(\Omega_{Z}, \Omega_{R}, \Omega_{\theta}\right)=\left(0,0, \Omega_{\theta}\right)$, où $\Omega_{\theta}=\frac{\partial U_{R}}{\partial z}-\frac{\partial U_{Z}}{\partial R}$ est représentée sur la figure 5 , gauche. Nous notons sur cette figure la présence de deux paires d'anneaux cohérents, créés par la circulation du fluide entre les jets d'injection, le plan central de stagnation, et le retour à travers les plaques poreuses. Ces anneaux correspondent aux zones de cisaillement pur, dans lesquelles la rotation et l'étirement coexistent, ayant la même valeur. Ces anneaux cohérents ne sont pas étirés, car la composante de la vitesse moyenne $\left\langle U_{\theta}\right\rangle$, mesurée à la périphérie des anneaux, est quasi-nulle.

Trois échelles sont à noter sur cette figure :

- l'échelle caractéristique de l'injection du fluide $d / 2$, où $d$ est le diamètre des jets $(d=1 \mathrm{~cm}, 263$ pixels $)$;

- l'échelle d'injection de l'enstrophie, $l=5 \mathrm{~mm}$, 130 pixels. C'est l'échelle caractéristique de l'écoulement, donnée par la largeur des anneaux cohérents; 

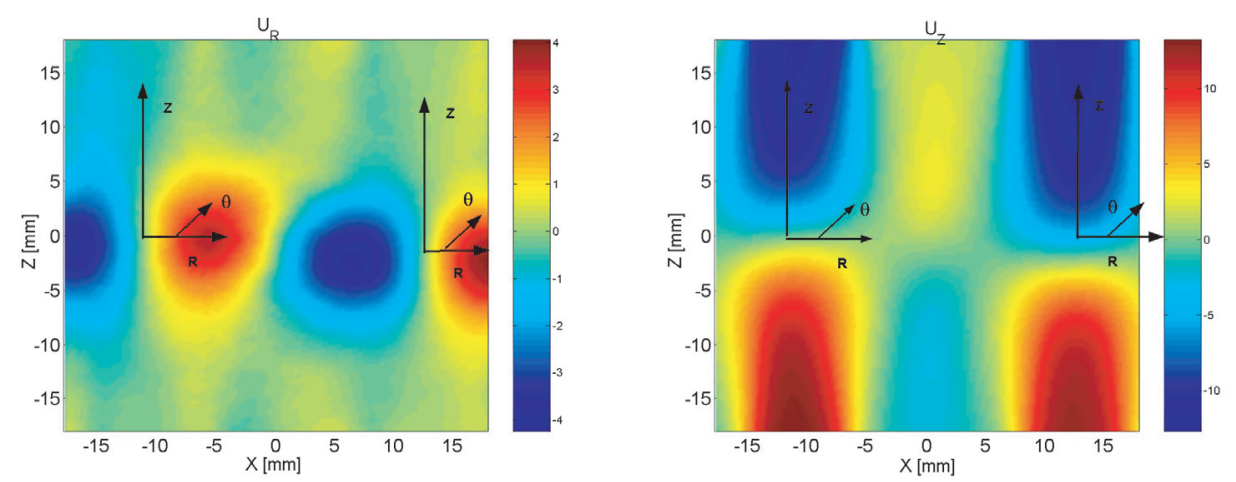

Fig. 2. Champ de vitesse moyenne : radiale $U_{R}$ (gauche) et verticale $U_{Z}$ (droite).
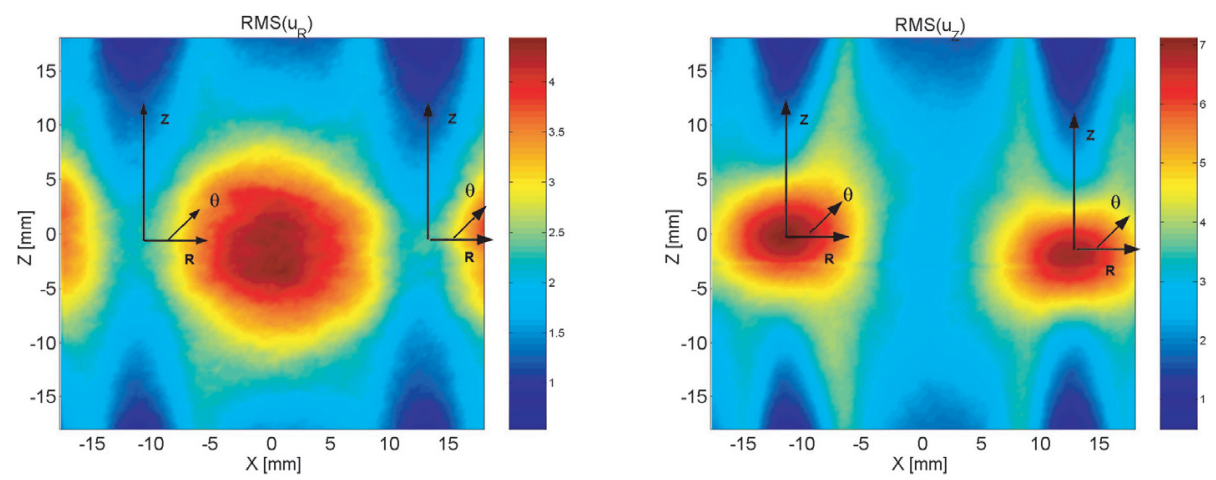

Fig. 3. $R M S\left(u_{R}\right)$ (gauche) et $R M S\left(u_{Z}\right)$ (droite).

- l'échelle (maximale) d'injection d'énergie. L'énergie est injectée sur une gamme d'échelle $[l, L]$, où $L=1,3 \mathrm{~cm}$, 350 pixels. Ceci est la distance entre l'axe du jet et le point de stagnation entre les jets. Évidemment, cette échelle est reliée au confinement du système et elle est donc l'échelle maximale de l'écoulement. À titre comparatif, la figure 5 (droite) représente la composante planaire de l'étirement du champ moyen, dans le plan $(X, Z)$. Nous observons que dans les anneaux cohérents, les valeurs de la vorticité et de l'étirement sont égales, comme dans le cas de la turbulence cisaillée pure. Les deux sont responsables de la dissipation d'énergie aux petites échelles. Dans la zone centrale du réacteur, la vorticité moyenne est nulle, alors que l'étirement est persistant, conduisant ainsi à une forte production de gradients de vitesse.

Dans les anneaux, la turbulence présente des propriétés localement homogènes (Fig. 6).

Il est connu que dans la région située entre des paires de tourbillons alternants, le fort cisaillement moyen conduit à de forts gradients de vitesse ou de scalaire [5]. Nous pouvons donc nous attendre à ce que la région centrale du réacteur, située entre les paires de structures tourbillonnaires, soit une zone de forte production de gradients et donc de forte dissipation et implicitement de forte enstrophie. La figure 7 illustre cette « abondance $»$ d'enstrophie dans la zone centrale du réacteur, et dans les régions occupées par les anneaux de vorticité. Nous pouvons donc par la suite décomposer l'écoulement en régions de forte rotation (et déformation, anneaux)

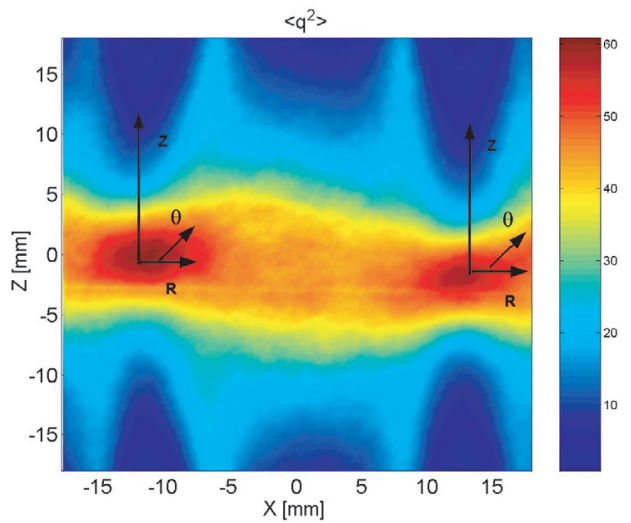

Fig. 4. Distribution de l'énergie totale : $\left\langle q^{2}\right\rangle=\left\langle u_{i} u_{i}\right\rangle=$ $2\left\langle u_{R}^{2}\right\rangle+\left\langle u_{Z}^{2}\right\rangle$.

et un écoulement plus calme (toutefois, turbulent). Notons aussi au passage que le rapport entre le temps caractéristique de l'écoulement moyen et le temps caractéristique de la turbulence est très faible $(\approx 0,1)$, ce qui correspond à une turbulence transportée en état gelé («baby $»$ turbulence).

\section{Résultats}

L'étape suivante consiste à analyser l'effet induit par ces régions de cisaillement sur la turbulence, en général. 

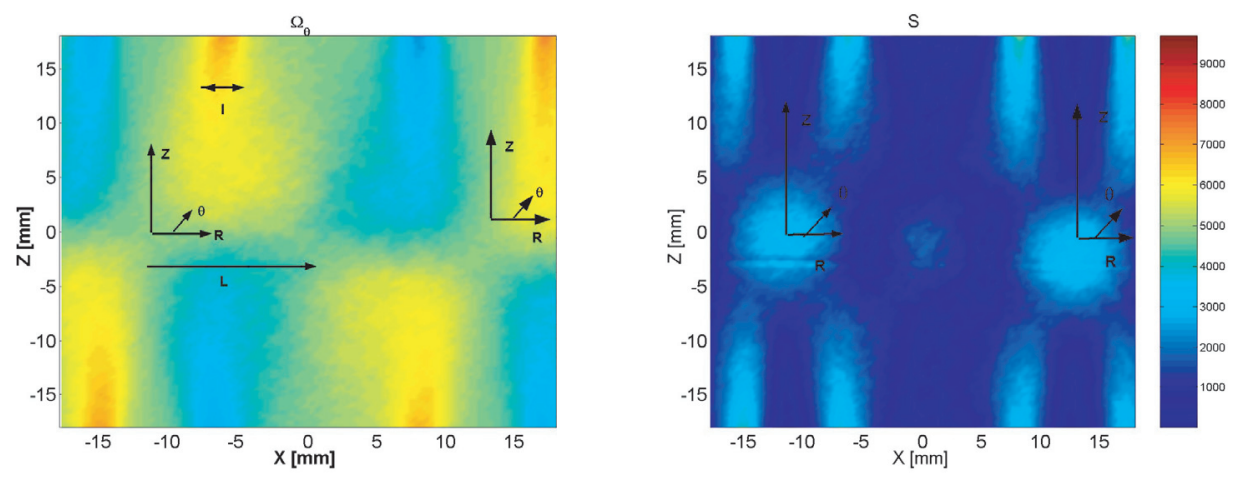

Fig. 5. Distribution de la composante azimutale de la vorticité du champ moyen $\Omega_{\theta}\left[\mathrm{s}^{-1}\right]$ (gauche) et de la composante planaire de l'étirement du champ moyen, $S$ (droite).
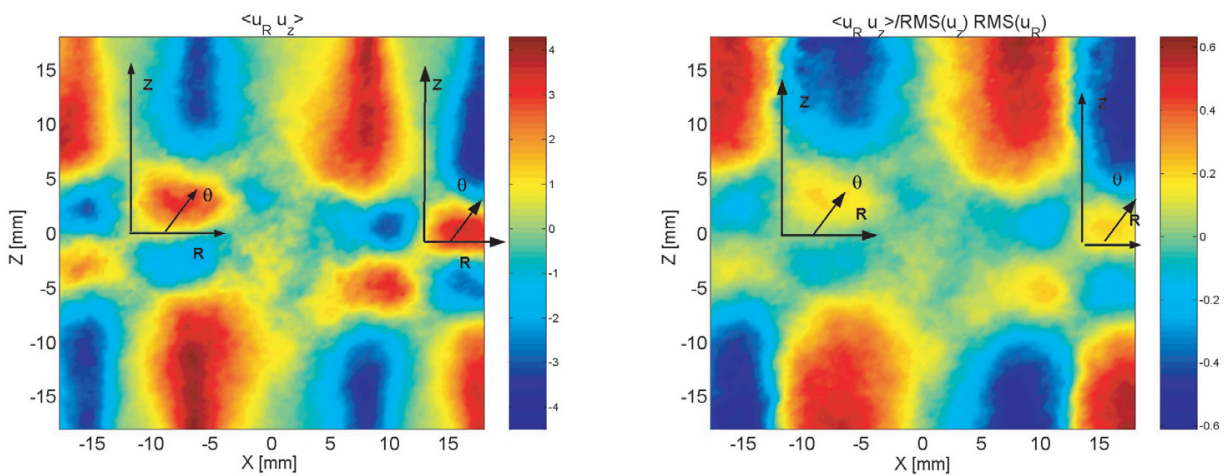

Fig. 6. Distribution de $\left\langle u_{R} u_{Z}\right\rangle$ (gauche) et sa valeur normalisée $\left\langle u_{R} u_{Z}\right\rangle / R M S\left(u_{R}\right) R M S\left(u_{z}\right)$.

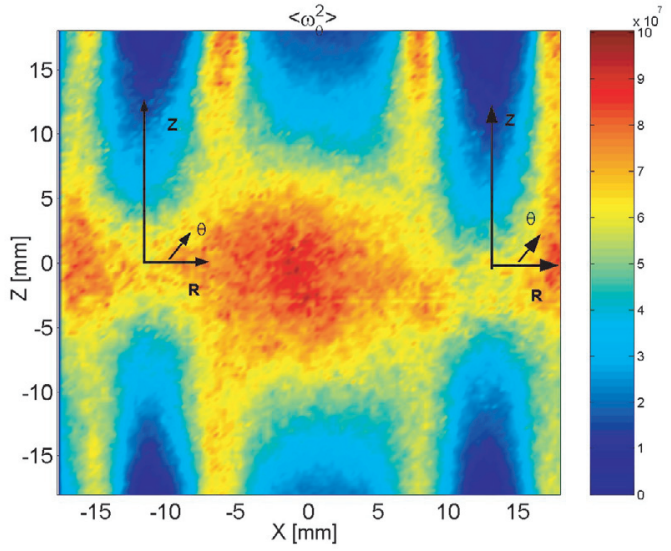

Fig. 7. Distribution de $R M S\left(\omega_{\theta}\right)$.

L'analyse spectrale (Sect. 3.1) sera suivie par un diagnostic du flux d'énergie et d'enstrophie à travers les échelles (Sect. 3.2). Une autre question adressée dans cet article est le scaling de l'enstrophie dans la zone inertielle (Sect. 3.3).

\subsection{Analyse spectrale}

La figure 8 illustre une coupe du spectre d'énergie (bidimensionnel), $E(k)$, en fonction du pseudo nombre

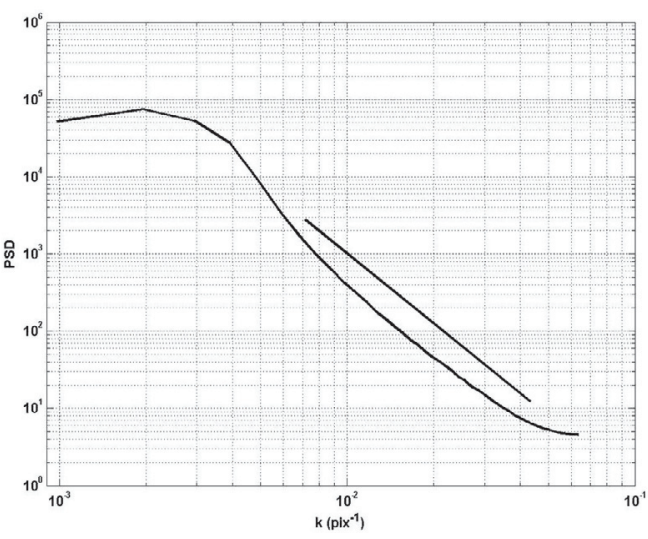

Fig. 8. Spectre d'énergie de la turbulence. La ligne continue représente la pente en $k^{-3}$.

d'onde $\left(k=\right.$ pixels $\left.^{-1}\right)$. Une large région pour laquelle $E(k) \sim k^{-3}$ est à noter, caractéristique de la turbulence en rotation (e.g. [6,7]). Une question ouverte subsiste sur une possible existence d'un régime en $-5 / 3$ au niveau des très petites échelles. À cause de la limite de résolution des mesures, ceci ne peut pas être détecté.

Notre écoulement est 3D et localement axisymétrique par rapport aux axes des jets. Pourtant, il est maintenant bien établi que la rotation conduit à des propriétés 
statistiques dans un plan perpendiculaire à l'axe de rotation, similaires à celles d'un écoulement turbulent 2D. Dans ce contexte, les deux régimes spectraux $(-3$ and $-5 / 3)$ correspondent respectivement à un régime d'enstrophie (dans lequel l'enstrophie cascade directement et la cascade d'énergie est inverse, [8]), et à un régime d'énergie. Ces deux régimes sont prédits théoriquement par [9] pour la turbulence 2D, et ils sont effectivement confirmés par quelques auteurs, e.g. [10], mais ils ne sont pas confirmés par la turbulence géostrophique [8,11].

Nous ne devons pas oublier que notre écoulement est $3 \mathrm{D}$, et que c'est la rotation locale qui induit une turbulence quasi-2D. Une analogie directe avec la turbulence $2 \mathrm{D}$ est à faire avec attention. Par conséquent, notre but suivant est de comprendre, fixer les limites et quantifier les différents régimes, en utilisant des grandeurs locales, i.e. les fonctions de structure.

Comme la vorticité est injectée dans l'écoulement sur une gamme d'échelles plus petites que $l$, et qu'elle cascade directement vers les échelles plus petites, nous analysons par la suite les fonctions de structure de vorticité. Si la cascade est directe et locale, alors un spectre en $k^{-\alpha}$ conduit à une énergie locale au nombre d'onde $k_{n}$ qui est $\sim \int_{k_{n}}^{k_{n+1}} k^{-\alpha} \mathrm{d} k \sim k^{-\alpha+1}$, et qui correspond dans l'espace réel (fonctions de structure d'ordre deux, ci-après $F S$ ) à $F S \sim r^{\alpha-1}=r^{2}$. Il est alors très simple de montrer que la loi d'échelle en $2 / 3$ pour les $F S$ correspond à un comportement en $-5 / 3$ dans l'espace de Fourier.

Par ailleurs, l'enstrophie des nombres d'onde $k_{n}$ est $\sim \int_{k_{n}}^{k_{n+1}} k^{2} k^{-\alpha} \mathrm{d} k \sim \int_{k_{n}}^{k_{n+1}} k^{-\alpha+2} \mathrm{~d} k$, et pour $\alpha=3$, elle devient $\sim \int_{k_{n}}^{k_{n+1}} k^{-1} \mathrm{~d} k \sim \log (k)$, ce qui conduit à une singularité dans l'espace réel, i.e. Log $(r)$. En conclusion, un spectre d'énergie en $k^{-3}$ conduit, pour une cascade d'enstrophie locale et directe, à des fonctions de structure de vorticité proportionnelles à $\log (r)$. Cette dernière affirmation sera étudiée plus profondement dans les soussections suivantes :

- nous allons d'abord montrer (Sect. 3.2) que la cascade d'enstrophie et d'énergie est directe pour des échelles plus petites que $l$, et inverse pour des échelles plus grandes que $l$;

- en utilisant des termes des grandes-échelles, nous étudions le comportement dans la ZI des FS de vorticité (Sect. 3.3).

\subsection{Cascade d'énergie et d'enstrophie directe et inverse}

Une analyse plus fine de la forme très abrupte du spectre d'énergie peut être obtenue en étudiant l'influence de la rotation sur les transferts énergétiques et d'enstrophie dans l'espace réel. Des statistiques des incréments de vitesse $\Delta u_{R}=u_{R}^{+}-u_{R}$ à travers la séparation $r=|\vec{r}|$, et les moments d'ordre deux de ces quantités $\left\langle\left(\Delta u_{R}\right)^{2}\right\rangle$ représentent l'énergie cinétique de l'écoulement à l'échelle en question, alors que les moments d'ordre trois $\left\langle\left(\Delta u_{R}\right)^{3}\right\rangle$ sont reliés au flux d'énergie moyen à l'échelle $r$. En particulier, son signe indique la direction du flux énergétique

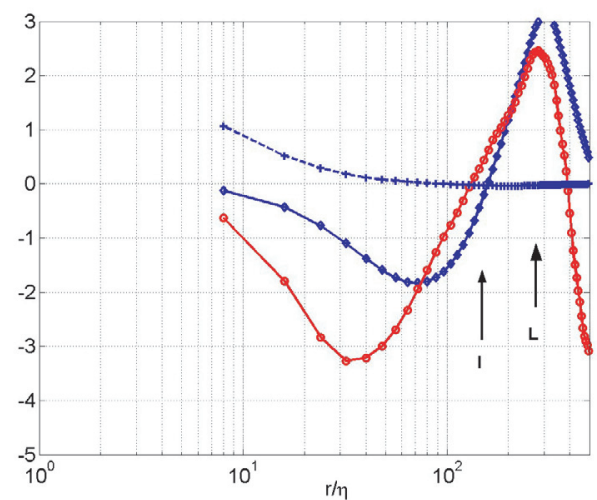

Fig. 9. Ligne continue et $\diamond$ : fonctions de structure d'ordre trois des incréments de vitesse, $\left\langle\left(\Delta u_{R}\right)^{3}\right\rangle$; ligne continue et $\circ$ : flux d'enstrophie $\left\langle\Delta u_{R}\left(\Delta \omega_{\theta}\right)^{2}\right\rangle ;+$ : corrélations de vorticité $\left\langle\omega_{\theta} \omega_{\theta}^{+}\right\rangle$.

à travers l'échelle $r$ : positif pour des transferts vers les échelles plus grandes et négatif pour des transferts vers les échelles plus petites.

Dans le cas de la turbulence en non-rotation à grands Reynolds, l'énergie est transférée à un taux constant pour toutes les échelles de la ZI, et $\left\langle\left(\Delta u_{R}\right)^{3}\right\rangle$ verifie la loi de Kolmogorov en $4 / 5$ : $\left\langle\left(\Delta u_{R}\right)^{3}\right\rangle=-4 / 5\langle\epsilon\rangle r$, où $\langle\epsilon\rangle$ est le taux moyen de dissipation de l'énergie cinétique. L'énergie se déplace, en moyenne, des grandes vers les petites échelles.

Pour la turbulence 2D, nous devons distinguer deux régimes possibles de cascade. Pour les échelles plus grandes que l'échelle d'injection, dans le régime de cascade inverse d'énergie, le transfert se fait en moyenne des petites vers les grandes échelles, et $\left\langle\left(\Delta u_{R}\right)^{3}\right\rangle$ est positif. Pour les échelles plus petites que l'échelle d'injection, dans le régime de cascade d'enstrophie, il n'y a pas de transfert d'énergie, et $\left\langle\left(\Delta u_{R}\right)^{3}\right\rangle$ devrait être égale à zéro.

Revenons maintenant à notre écoulement. Afin de mieux comprendre la direction selon laquelle l'énergie cascade, nous calculons par la suite les $F S$ de $3^{\mathrm{e}}$ ordre, i.e. $\left\langle\left(\Delta u_{R}\right)^{3}\right\rangle$, représentées sur la figure 9. Ce calcul est fait pour la totalité de l'image. De nouveau, nous voyons le rôle crucial joué par l'échelle $l$. Pour des échelles plus petites que $l,\left\langle\left(\Delta u_{R}\right)^{3}\right\rangle<0$ comme c'est le cas en turbulence $3 \mathrm{D}$, et l'énergie est transférée vers les échelles plus petites où elle est dissipée. Une zone de proportionnalité avec $r$ est à noter, comme prédit par Kolmogorov. Pour des échelles plus grandes que $l$, le transfert d'énergie est positif, vers les échelles plus grandes, suggérant ainsi qu'une cascade d'énergie inverse commence à se développer aux grandes échelles. Pour ces échelles grandes, le système se comporte comme une turbulence en rotation (quasi-2D). Des observations similaires ont été reportées par [12], à partir des mesures en 1-point au voisinage d'un tourbillon intense.

Des résultats similaires sont obtenus pour la cascade d'enstrophie, qui est directe sur une grande gamme d'échelles (voir Fig. 9), en complet accord avec l'image fournie par les spectres. 


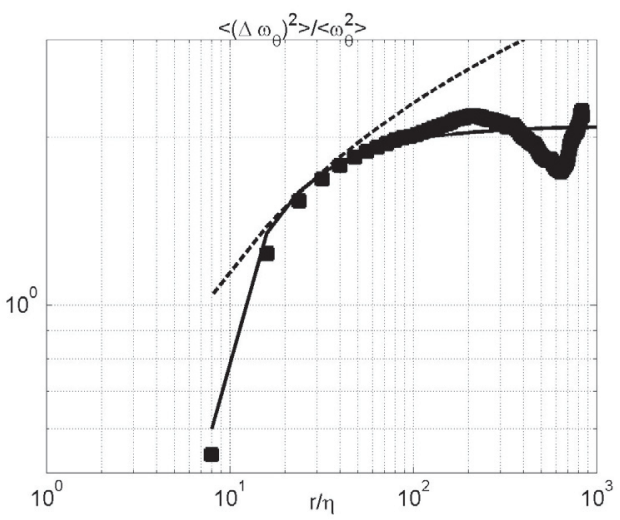

Fig. 10. Fonctions de structure normalisées de la vorticité azimutale $(\square):\left\langle\left(\Delta \omega_{\theta}\right)^{2}\right\rangle /\left\langle\omega_{\theta}^{2}\right\rangle$. La ligne pointillée représente $\sim \log (r)$. La ligne continue représente le modèle $a+b \frac{1}{r}$.

\subsection{Fonctions de structure d'ordre deux de vorticité}

Dans le cadre de la théorie de Kolmogorov, les corrélations de vorticité en deux points devraient décroître comme $r^{-4 / 3}$ dans la zone inertielle. Pourtant, cette loi d'échelles n'a jamais été obtenue à partir des données expérimentales ou des DNS, dans une turbulence 2D ou 3D. Il a été montré que les fonctions de structure d'ordre deux de vorticité (pour n'importe laquelle de ses trois composantes) ne présentent pas de lois d'échelles (voir par exemple la figure 1 de [13]).

Les fonctions de structure de second-ordre de la vorticité (azimuthale) sont représentées sur la figure 10. Elles sont normalisées par la variance de vorticité, donc la limite aux grandes échelles est de 2. Deux conclusions sont à tirer sur cette figure. La première est que la longueur de corrélation de la vorticité azimuthale est très grande, et elle est égale à $l$, la largeur des anneaux cohérents de vorticité. Nous reportons ici des statistiques qui sont très différentes de celles usuellement reportées dans la littérature pour la turbulence 3D (e.g. [13]). Notre approche originale est de créer expérimentalement des zones de forte rotation, à l'intérieur desquelles la turbulence présente des propriétés 3D. La seconde propriété des SF2 de vorticité est qu'elles présentent réellement une zone pour laquelle le comportement en échelles est de $\sim \log (r)$.

Le comportement logarithmique a été également prédit par [1], dans le contexte de la turbulence strictement 2D. A notre connaissance, cette loi n'a pas été confirmée directement. Quelques conséquences de la loi Log ont été reportées par [10]. Par la suite, nous montrons analytiquement que le comportement en Log $(r)$ est valide dans notre écoulement.

En 3D, l'équation de transport (en coordonnées cartesiennes) de l'enstrophie est [13] :

$$
\left\langle\Delta u_{1}\left(\Delta \omega_{i}\right)^{2}\right\rangle-2 \nu \frac{\partial}{\partial r}\left\langle\left(\Delta \omega_{i}\right)^{2}\right\rangle=-2\left\langle\omega_{i} \omega_{1}^{+} u_{i}^{+}-\omega_{i}^{+} \omega_{1} u_{i}\right\rangle
$$

Cette équation a été testée en utilisant des données expérimentales dans un sillage [13]. Des mesures avec une sonde mesurant toutes les composantes de la vorticité ont montré que ces termes sont non-nuls pour des échelles plus petites que $20 \eta$, avec un maximum à $5 \eta$, ainsi soulignant la nature petites-échelles de la vorticité. D'une manière intuitive, ceci est en accord avec la présence de filaments de vorticité très intenses, avec un diamètre de $4-8 \eta$. Nous insistons ici sur la différence entre notre écoulement et d'autres écoulements classiques. Dans notre écoulement, les anneaux de vorticité «vivant » aux grandes échelles conduisent à des corrélations de vorticité aussi grandes que la taille des anneaux.

Retournons maintenant aux calculs. En adoptant la procédure indiquée dans [1], nous passons dans le système de référence local, et en introduisant le champ de vitesse lagrangien $\vec{v}(t, \vec{x})$, relié à la vitesse eulerienne : $\vec{u}(t, \vec{x})=v\left(t, \vec{x}-\int_{0}^{t} \vec{v}(\tau, 0)\right) \mathrm{d} \tau$. L'équation de transport lagrangien de l'enstrophie devient ainsi (en négligeant les effets visqueux) :

$$
\frac{D}{D t}\left\langle\left(\Delta \omega_{i}\right)^{2}\right\rangle=\mathcal{F}(r)
$$

avec $\mathcal{F}(r)=-2 \frac{\partial}{\partial r_{\alpha}}\left\langle\omega_{i} \omega_{\alpha}^{+} u_{i}^{+}-\omega_{i}^{+} \omega_{\alpha} u_{i}\right\rangle$. Dans un premier temps, nous considérons dans le terme de forçage uniquement la composante quasi-continue, agissant aux échelles $r>L$, et en omettant la composante fluctuante du terme de forçage. Nous allons ainsi décrire les corrélations d'enstrophie transportées par un champ de vitesse grandeséchelles, qui est écrit par la suite en utilisant l'axisymétrie autour de la direction d'injection $Z(\partial / \partial \theta\langle\rangle \equiv 0)$ et l'isotropie des petites échelles :

$$
\begin{aligned}
\mathcal{F}(r) & \simeq-2\left[\frac{1}{r}+\frac{\partial}{\partial r}\right]\left\langle\Omega_{\theta} \omega_{r}^{+} u_{\theta}^{+}-\Omega_{\theta}^{+} \omega_{r} u_{\theta}\right\rangle \\
& =-2\left[\frac{1}{r}+\frac{\partial}{\partial r}\right]\left[\Omega_{\theta}\left\langle\omega_{r}^{+} u_{\theta}^{+}\right\rangle-\Omega_{\theta}^{+}\left\langle\omega_{r} u_{\theta}\right\rangle\right] \\
& \simeq-2\left[\frac{1}{r}+\frac{\partial}{\partial r}\right]\left[\Omega_{\theta}\left\langle\omega_{\theta}^{+} u_{r}^{+}\right\rangle-\Omega_{\theta}^{+}\left\langle\omega_{\theta} u_{r}\right\rangle\right]
\end{aligned}
$$

Avec ces approximations raisonnables, $\mathcal{F}(r)$ peut être calculée facilement à partir de nos mesures $2 \mathrm{D}$ de champ de vitesse. Le comportement de ce terme de forçage, qui n'est pas montré ici, est plutôt concentré aux grandes échelles, plus grandes que la zone inertielle, à laquelle nous nous intéressons. Ce comportement est également particulier à notre écoulement, pour lequel la vorticité moyenne et l'étirement moyen sont présents aux grandes échelles. Ce terme est ainsi négligeable pour les petites échelles. Dans le domaine des grandes échelles, il se comporte comme $\mathcal{F} \approx\left[\frac{1}{r}+\frac{\partial}{\partial r}\right] \log (r) \simeq \frac{1}{r}+\frac{\log (r)}{r}$

Dans le contexte de l'isotropie locale, l'enstrophie totale peut être écrite comme :

$$
\begin{aligned}
\left\langle\left(\Delta \omega_{i}\right)^{2}\right\rangle & =\left\langle\left(\Delta \omega_{r}\right)^{2}\right\rangle+2\left[1+\frac{r}{2} \frac{\partial}{\partial r}\right]\left\langle\left(\Delta \omega_{r}\right)^{2}\right\rangle \\
& =\left[3+r \frac{\partial}{\partial r}\right]\left\langle\left(\Delta \omega_{r}\right)^{2}\right\rangle
\end{aligned}
$$

La relation entre le temps et la distance reste, dans notre écoulement, comme dans le cas $2 \mathrm{D}: r \exp (S t)=L$, où $S$ 
est l'étirement moyen du champ de vitesse dans le plan $X, Z$. Notons ici que cette hypothèse est uniquement valable dans le cas de notre écoulement axisymétrique. Avec ces considérations, nous allons de plus supposer que $\mathcal{F}(r)$ agit uniquement pour des échelles plus grandes que $L$, et qu'elle est constante à ces échelles. C'est la procédure abordée par [1]. Ainsi, l'équation (2) conduit à

$$
\left\langle\left(\Delta \omega_{i}\right)^{2}\right\rangle \simeq \tau^{*} \cdot C \simeq C \frac{1}{S} \log \left(\frac{L}{r}\right)=C_{1}+C_{2} \log (r)
$$

Ici, $C, C_{1}$ et $C_{2}$ sont des constantes et nous sommes uniquement intéressés dans les lois d'échelles de la ZI.

Notre équation (5) est équivalente avec l'équation (11) de [1]. Le temps $\tau^{*}$ est le temps nécessaire à la distance moyenne entre deux points pour croître de $r$ à $L$. La solution de $(5)$ conduit à $\left\langle\left(\Delta \omega_{i}\right)^{2}\right\rangle \propto a+b \log (r)$ et finalement

$$
\left\langle\left(\Delta \omega_{\theta}\right)^{2}\right\rangle \propto a+b \log (r)
$$

avec $a$ et $b$ constantes. Le comportement prédit par notre relation (6) est partiellement vérifié par les données expérimentales, comme il est montré sur la figure 10. La relation (6) correspond à un spectre énergétique $E(k) \propto k^{-3}$. Une meilleure description du comportement en échelles des fonctions de structure d'enstrophie est obtenue en tenant compte du forçage d'enstrophie à chaque échelle. Ce travail n'est pas reporté ici.

\section{Conclusions}

Nous avons montré que la création de structures tourbillonnaires annulaires conduit à une structure particulière de la turbulence aux petites échelles. Le résultat le plus important est que le champ de vorticité présente une longueur de corrélation qui est aussi grande que la taille des anneaux de vorticité. Les grandes échelles de l'écoulement présentent des propriétés similaires à la turbulence quasi-2D (cascade d'énergie et d'enstrophie inverses), alors que les échelles plus petites sont 3D (cascades d'énergie et d'enstrophie directes). En considérant l'effet des grandes échelles, i.e. de la vorticité moyenne, sur le champ de vorticité, il est possible d'expliquer le comportement dans la ZI des fonctions de structure d'ordre deux, ainsi que leur variation en échelles. Cette question est importante afin de mieux comprendre et quantifier l'intermittence aux petites échelles des écoulements turbulents.

\section{Références}

[1] G. Falkovich, V. Lebedev, Nonlocal vorticity cascade in two dimensions, Phys. Rev. E 49 (1994) 1800-1803

[2] B. Sahr, M. Birouk, I. Gökalp, An attempt to realize experimental isotropic turbulence at low reynolds numbers, Flow, Turbulence and Combustion 70 (2003) 325-348

[3] W. Hwang, J.K. Eaton, Creating an homogeneous and isotropic turbulence without a mean flow, Exp. Fluids (2003) online

[4] J.F. Krawczynski, B. Renou, L. Danaila, F.X. Demoulin Small-scale measurements in a partially stirred reactor (pasr), Exp. Fluids 40 (2006) 667-682

[5] S. Goto, S. Kida, Enhanced stretching of material lines by antiparallel vortex pairs in turbulence, Fluid Dynamics Research 33 (2003) 403-431

[6] M. Hossain, Inverse energy cascades in three dimensional turbulence, Phys. Fluids B 3 (1991) 511-514

[7] M. Hossain, Reduction of the dimensionality of turbulence due to a strong rotation, Phys. Fluids 6 (1994) 1077-1080

[8] S.D. Danilov, D. Gurarie, Quasi-two-dimensional turbulence, Physics-Uspekhi 43 (2000) 863

[9] R.H. Kraichnan, Inertial-range transfer in two-and three-dimensional turbulence, J. Fluid Mech. 47 (1971) 525

[10] J. Paret, M.-C. Jullien, P. Tabeling, Vorticity statistics in the two-dimensional enstrophy cascade, Phys. Rev. Lett. 83 (1999) 3418

[11] E. Lindborg, Can the atmospheric kinetic energy spectrum be explained by two-dimensional turbulence? J. Fluid Mech. 388 (1999) 259

[12] F. Chillà, C. Simand, J.F. Pinton, Inhomogeneous turbulence in the vicinity of a large-scale coherent vortex, Europhys. Lett. 49 (2000) 336

[13] R.A. Antonia, M. Ould-Rouis, Y. Zhu, F. Anselmet, Transport of turbulent vorticity increments, Phys. Rev. E 57 (1998) 5483 